\title{
Mapeamento Sistemático sobre Abordagens de Mensuração de Autorregulação da Aprendizagem
}

\author{
Rodrigo Lins Rodrigues - UFRPE, rodrigo.linsrodrigues@ufrpe.br \\ Jorge Luis Cavalcanti Ramos - UNIVASF, jorgeluiscavalcanti@ univasf.edu.br \\ João Carlos Sedraz Silva - UNIVASF, joao.sedraz@univasf.edu.br \\ Hugo Vieira Lucena de Souza - UFPE, hvlsouza@gmail.com \\ Alex Sandro Gomes - UPE, asg@ cin.ufpe.br \\ Fernando da Fonseca de Souza - UFPE, fdfd@cin.ufpe.br
}

\begin{abstract}
Resumo. Este trabalho teve como objetivo o desenvolvimento de um mapeamento sistemático com a finalidade de identificar as principais abordagens de mensuração de características de autorregulação da aprendizagem em ambientes online. A identificação destas abordagens fornecem subsídios para identificar as principais formas de análises e coletas que estão sendo utilizadas na literatura. Os resultados mostram que ainda é pouca expressiva a quantidade de trabalhos que utilizam dados comportamentais, através de logs, para a mensuração em tempo real. Este parece ser um tema desafiador especialmente considerando o número cada vez maior de cursos a distância, número de alunos e volume de dados gerados em plataformas de aprendizagem online.
\end{abstract}

Palavras-chave. Autorregulação da Aprendizagem, Mapeamento Sistemático, Sistema de Gestão da Aprendizagem.

\section{Systematic mapping about approaches measurement of Self- Regulation Learning}

Abstract. This study aimed to develop a systematic mapping in order to identify the main approaches to measuring learning self-regulation characteristics in online environments. The identification of these approaches provides subsidies to identify the main forms of analysis and collections that are being used in the literature. The results show that it is still the little significant amount of work using behavioral data, via logs, to measure in real time. This seems to be a challenging issue especially considering the increasing number of distance learning courses, number of students and amount of data generated in online learning platforms.

Keywords. Self-Regulated Learning, Systematic Mapping, Learning Management System.

\section{Introdução}

Em cursos promovidos por meio de ambientes virtuais de aprendizagem, o acompanhamento é dificultado pela falta meios de comunicação entre professores e alunos e pela falta de percepção e análise de aspectos comportamentais da interação dos usuários (WANG, DOLL, et al., 2013). Analisar e monitorar esse volume de interações entre professor, aprendizes e conteúdo é uma importante e complexa tarefa do professor no processo de aprendizagem online. 
O estudante nesta modalidade é tido como elemento central do processo de aprendizagem. Estes muitas vezes deparam-se com a flexibilidade de tempo, local e recursos dos ambientes virtuais de aprendizagem, quando é exigido um maior nível de responsabilidade do aluno, que passa a ter controle sobre o seu próprio processo de aprendizagem (PINTRICH, 1999).

Estudos vem sendo desenvolvidos e autores como (CHEN e PAUL, 2003; SALAS et al., 2002) e (Roll \& Winne, 2015) afirmam que diferenças no comportamento individual de estudantes interferem na aprendizagem a distância. Estes estudos buscam identificar variáveis individuais que afetam os resultados da aprendizagem de acordo com o perfil comportamental de cada estudante nas plataformas de aprendizagem.

O fenômeno comportamental de autorregulação da aprendizagem (SRL) é tema de estudo dentro da Psicologia Cognitiva. Um dos aspectos estudados é a estrutura de estratégias de gestão de recursos, gestão do tempo, do esforço, do ambiente e da interação e busca por ajuda de outras pessoas (CHEN, 2002; PINTRICH, 1999; ZIMMERMAN e MARTINEZ-PONS, 1988).

Existem múltiplas formas de estabelecer estratégias de autorregulação da aprendizagem. Estudantes que possuem estas habilidades conseguem controlar o ritmo, o direcionamento do processo de aprendizagem e gerenciar um conjunto de recursos do ambiente de aprendizagem, realizando escolhas que podem auxiliar o desenvolvimento de sua estrutura cognitiva (CHEN e PAUL, 2003); (BROADBENT e POON, 2015) .

Com o objetivo de colocar em perspectiva uma ampla coleção desses estudos, assim como também reunir estudos que tragam relatos sobre possibilidades de mensuração quantitativa de características de autorregulação que ocorrem com estudantes, este trabalho visou realizar um mapeamento sistemático da literatura com o objetivo de identificar um arcabouço de pesquisas e resultados que busquem mensurar as características de autorregulação da aprendizagem em ambientes online.

\section{Características da autorregulação da aprendizagem em ambientes de online}

Alguns pesquisadores acreditam que os ambientes de aprendizagem on-line exigem do aluno uma maior responsabilidade para o processo de aprendizagem (DABBAGH e KITSANTAS, 2005); (SCHUNK e ZIMMERMAN, 1998).

Estes autores acreditam que as habilidades de autorregulação são essenciais para o sucesso em situações de aprendizagem autônomas e que o desenvolvimento dessas habilidades pode ser suportado por ferramentas pedagógicas baseadas na Web (AZEVEDO, 2005); (DABBAGH e KITSANTAS, 2004);

Estas habilidades podem ser importantes para alunos que participam de cursos nesta modalidade (DABBAGH e KITSANTAS, 2004); (GARRISON, 2003); (HARTLEY e BENDIXEN, 2001); (SCHUNK e ZIMMERMAN, 1998). Por exemplo, Dabbagh e Kitsantas (2004) e (Barnard, Lan, To, \& Paton, 2009) argumentaram que em um ambiente de aprendizagem baseado na Web, os alunos devem possuir um alto grau de competência de autorregulação para gerir seus objetivos de aprendizagem, enquanto que em ambientes tradicionais face-a-face em sala de aula, o instrutor exerce um controle significativo sobre o processo de aprendizagem e é capaz de monitorar a atenção do aluno, seu engajamento e o seu progresso. Da mesma forma, autores como Kinzie (1990); (Adesope, Zhou, \& Nesbit, 2015) afirmam que a habilidades de autorregulação é um dos três requisitos críticos para o sucesso do aluno. A utilização eficaz das estratégias de autorregulação da aprendizagem é essencial em situações de 
aprendizagem flexíveis, devido ao alto grau de autonomia exigida do aluno na modalidade a distância, consequência da ausência física do professor.

Ainda é pouco expressiva a quantidade de pesquisas sobre as características de autorregulação da aprendizagem com foco em ambientes de EAD. A maioria dos instrumentos de coleta disponibilizados na literatura para a mensuração de SRL foram desenvolvidos para ambientes presenciais de ensino. Na seção a seguir explicitamos, o método de mapeamento sistemático da literatura utilizado para identificar as diferentes abordagens mensuração que vem sendo desenvolvidas ao longo dos anos, bem como as diversas formas de coleta que foram utilizadas.

\section{Método}

O estudo de Mapeamento Sistemático (MS) é um método projetado para fornecer uma ampla visão de uma determinada área de pesquisa, que permite identificar, quantificar e analisar os resultados, estabelecendo evidências da mesma sobre um determinado tema (Kitchenham, 2007), (Arksey \& O’Malley, 2005).

$\mathrm{Na}$ primeira etapa destes estudo foi realizada a concepção de um protocolo, no qual foram descritos o processo e os métodos aplicados na pesquisa bibliográfica. As informações que fazem parte do protocolo serão apresentadas nas subseções seguintes.

Esta etapa teve o objetivo de identificar, catalogar, e classificar os métodos de análise de dados utilizados para mensurar características de autorregulação da aprendizagem em ambientes de LMS, com o intuito de identificar lacunas de pesquisa que subsidie e reforce a questão de uma pesquisa em andamento.

\subsubsection{Questões de pesquisa}

Uma das etapas essenciais do Mapeamento Sistemático é a definição das questões de pesquisa que conduzem a busca dos documentos relevantes, permitindo posteriormente a triagem de documentos, keywording de abstracts e extração dos dados (Petersen, 2007). Partindo do objetivo para responder a questão central do estudo que é: "Quais os principais métodos utilizados na literatura para a mensuração das características da autorregulação da aprendizagem em ambientes de LMS?’.

Foram elaboradas quatro questões secundárias que tiveram como intuito auxiliar as seguintes respostas:

- Q1: Qual a natureza das principais pesquisas que vem sendo desenvolvidas com o tema de Autorregulação da Aprendizagem?

- Q2: Quais as principais formas de coleta, no que diz respeito aos construtos de SRL, em ambientes de LMS?

- Q3: Quais as principais técnicas estão sendo utilizadas para analisar e mensurar as características de SRL?

- Q4: Quais as principais abordagens de pesquisa que dedicam esforços para o desenvolvimento e aplicação de técnicas de mensuração de SRL?

Estas questões serviram de direcionamento para a elaboração de uma string de busca que será descrita na próxima subseção.

\subsubsection{Critérios de Inclusão e Exclusão}

Para a inclusão de um trabalho na pesquisa foi determinada sua relevância em relação às questões de investigação através da análise do título, palavras-chave e resumo. Dois critérios de inclusão foram levados em consideração nessa análise: (1) o primeiro é que 
o trabalho deveria ter explicitamente o método/técnica que foi utilizado para mensurar características de autorregulação da aprendizagem e o segundo (2) que o trabalho apresentasse uma abordagem de mensuração com foco em ambientes de LMS. Trabalhos que não apresentaram tais características foram excluídos no processo de seleção. Na busca retornada nesta segunda string foram retirados apenas dois trabalhos.

\subsubsection{Estratégia de busca}

A fonte de pesquisa utilizada para a busca dos estudos primários foi a base Elsevier Scopus (http://www.scopus.com), uma vez que este repositório também inclui as bases da IEEE Digital Library, ACM Digital Library e ScienceDirect. Para a construção dos termos chaves da pesquisa foram utilizadas as palavras-chave da questão central.

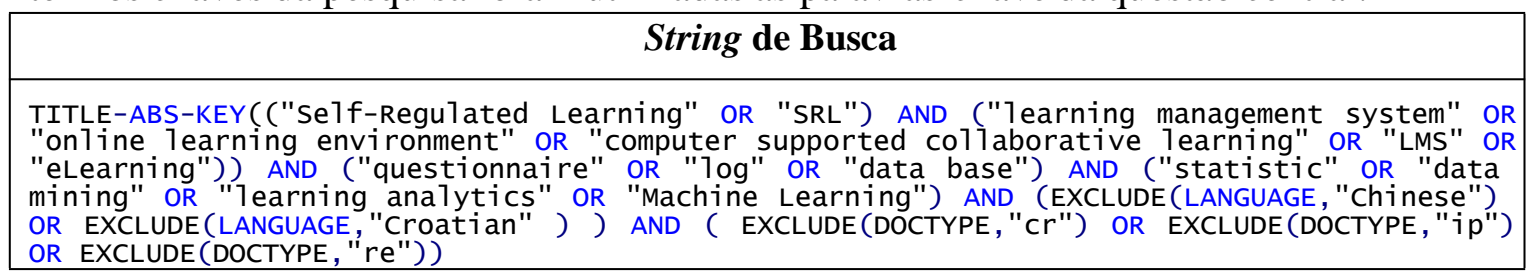

Nesta segunda string de busca tivemos como resultado 18 trabalhos primários. Dos 75 trabalhos encontrados na primeira string de busca, apenas 18 trabalhos utilizaram algum tipo de abordagem quantitativa para mensurar construtos de autorregulação da aprendizagem.

É importante mencionar que nesta segunda string de busca, a pesquisa foi complementada com sete artigos de uma série especial do Journal of Learning Analytics sobre 'Self-regulated learning and learning analytics' e mais dois trabalhos, um do periódico Lecture Notes in Computer Science e outro trabalho da International Conference on Educational Data Mining.

Os trabalhos foram distribuídos por ordem cronológica de publicações nos últimos oito anos. É interessante observarmos que o ano de 2015 , foi o ano que obteve um maior número de trabalhos na área.

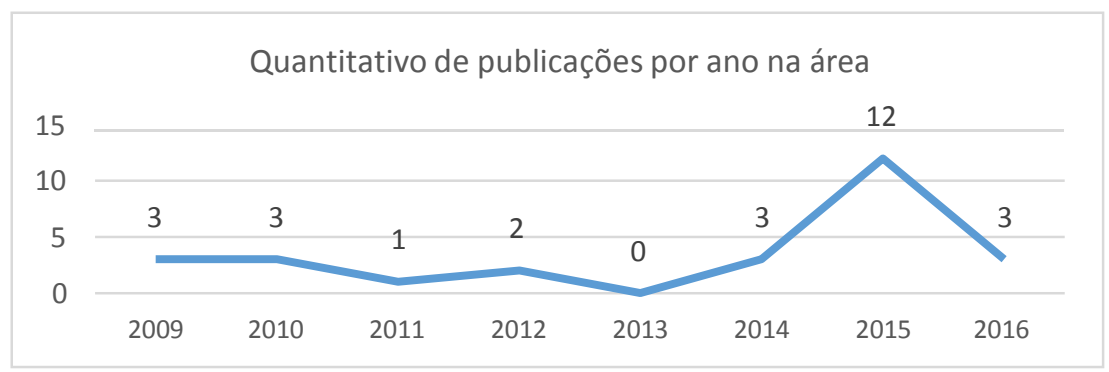

Figura 3: Quantidade de publicações na área nos últimos seis anos.

Dentre todos os trabalhos, $22(91,66 \%)$ foram artigos em periódicos e apenas 2 $(8,3 \%)$ artigos publicados em conferências.

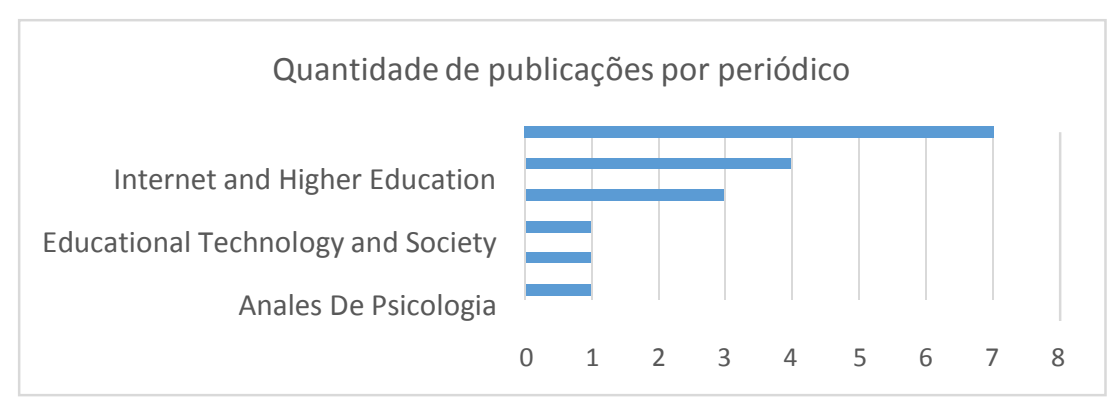


Figura 1: Principais periódicos com publicações na área nos últimos seis anos.

O periódico que teve maior quantidade de publicações na área foi o Journal of Learning Analytics com sete publicações seguido pelos periódicos Internet and Higher Education e o Computers in Human Behavior com um total de sete publicações.

\section{Análise e Interpretação dos Resultados}

O processo foi iniciado com a leitura dos vinte e quatro artigos selecionados na segunda interação. O processo de análise iniciou-se com a compreensão dos objetivos e das metodologias de cada um dos artigos, selecionando informações relevantes para responder cada uma das questões de pesquisa.

\subsection{Primeira questão de pesquisa}

A primeira questão de pesquisa focou na identificação da natureza das pesquisas que vem sendo desenvolvidas na área. Estas pesquisas tiveram como temas principais a aprendizagem colaborativa autorregulada, como pode ser visto nos trabalhos (Gašević, Adesope, Joksimović, \& Kovanović, 2015), (Malmberg, Järvelä, Järvenoja, \& Panadero, 2015), (Panadero, Kirschner, Järvelä, Malmberg, \& Järvenoja, 2015) e no estudo das relações entre autorregulação da aprendizagem e desempenho acadêmico, dentro os quais os principais trabalhos foram (Park \& Lim, 2015), (Ali, Hatala, Gašević, \& Winne, 2014), (Bondareva, et al., 2013).

Uma característica interessante das pesquisas que trabalharam com análise de dados, especificamente com foco na previsibilidade do desempenho acadêmico de alunos, foram realizadas através de abordagens de coleta com questionários, nos quais foram utilizados questionários previamente validados na literatura, tais como os MSLQ e OSLQ para a realização de inferências sobre o desempenho de estudantes, descritos a seguir.

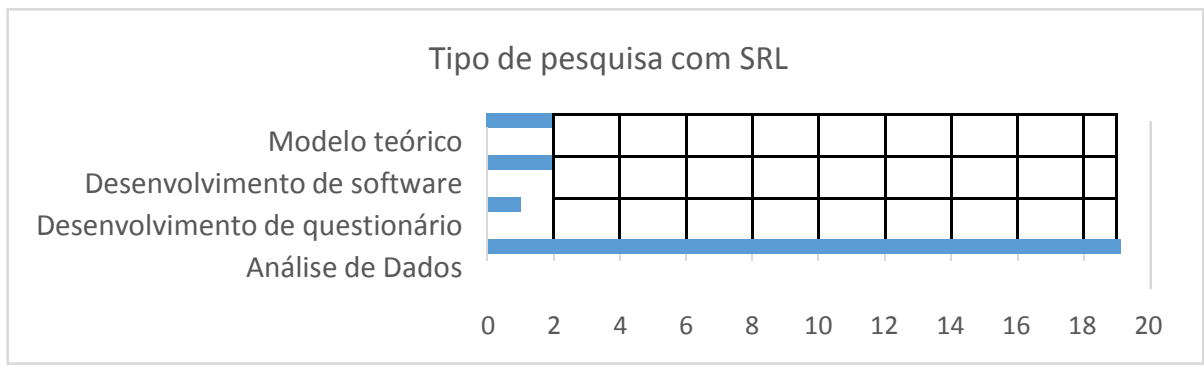

Figura 2: Tipo de pesquisa em SRL.

Os três outros tipos de abordagens encontradas na literatura foram: a validação de modelos teóricos de SRL, o desenvolvimento de software para suporte a SRL e o desenvolvimento de questionário.

Os artigos sobre validação de modelos teóricos de SRL (Roll \& Winne, 2015), (Sanchez-Santillan, Paule-Ruiz, Cerezo, \& Alvarez-García, 2016) buscaram entender o estado atual da investigação de SRL, buscando identificar temas e desafios de apoio a SRL em ambientes de LMS. Apesar destes artigos não aplicarem nenhuma técnica de mensuração, foram considerados nesta pesquisa pois citam diversos artigos que utilizam.

Dentre os artigos que propuseram o desenvolvimento de software, um que merece destaque é o artigo desenvolvido por (Nussbaumer, Hillemann, Gütl, \& Albert, 2015). O autor apresenta uma abordagem conceitual de serviço baseado na Web para apoio à aprendizagem autorregulada em ambientes virtuais. $\mathrm{O}$ trabalho fornece uma 
descrição da implementação técnica do modelo conceitual e uma validação de usabilidade com usuários de graduação.

Em relação aos trabalhos que se propuseram a desenvolver questionário específico para mensurar SRL, há o trabalho (Barnard, Lan, To, \& Paton, 2009). A pesquisa é justificada pela ausência de um instrumento de coleta que aborde os construtos de SRL especificamente para aprendizagem online, no qual é realizado a construção de um instrumento com vinte e quatro questões que abordam seis construtos, tais como: estruturação do ambiente, busca por ajuda, estratégias para realização da tarefa, auto avaliação, gerenciamento do tempo e definição de metas. Estes construtos foram validados através de análise fatorial confirmatória que buscou avaliar as propriedades psicométricas, por meio da qual os resultados indicam que o instrumento OSLQ é uma medida aceitável de autorregulação nos ambientes de aprendizagem online.

\subsection{Segunda questão de pesquisa}

A segunda questão de pesquisa desenvolvida neste trabalho: quais as principais formas de coleta, no que diz respeito aos construtos de SRL, em ambientes de LMS? Os resultados encontrados nesta segunda questão de pesquisa mostraram que a grande maioria dos trabalhos que se propõem a mensurar SRL utilizam questionários.

Dos onze trabalhos que utilizaram esta abordagem, seis deles utilizaram o questionário MSLQ (Yukselturk \& Bulut, 2009), (Adesope, Zhou, \& Nesbit, 2015), (Panadero, Kirschner, Järvelä, Malmberg, \& Järvenoja, 2015), (Sanchez-Santillan, Paule-Ruiz, Cerezo, \& Alvarez-García, 2016), (Ali, Hatala, Gašević, \& Winne, 2014). Este é o questionário mais utilizado na literatura, embora tenha sido construído para ser utilizado em ensino presencial.

Outro instrumento que foi utilizado nas pesquisas com questionário foi o OSLQ, um questionário desenvolvido por (Barnard, Lan, To, \& Paton, 2009) com foco em características de SRL em ambientes de LMS.

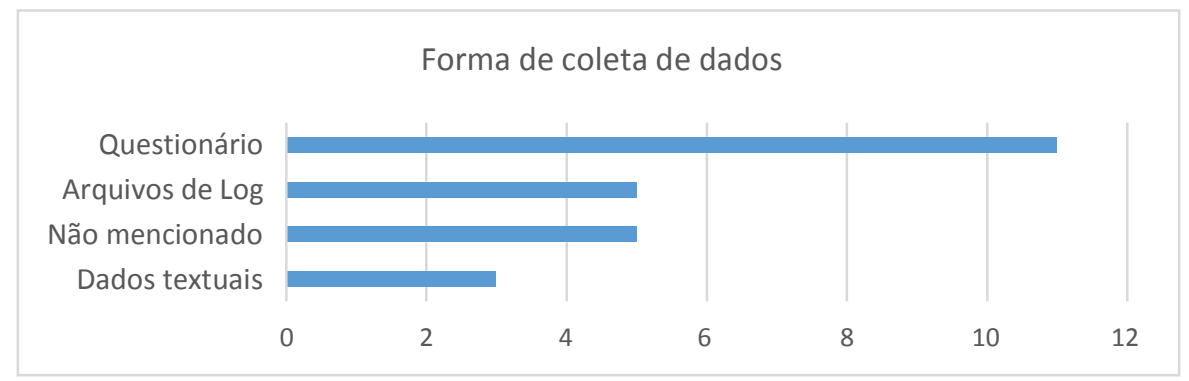

Figura 3: Forma de coleta de dados.

Uma segunda abordagem de coleta de dados utilizada nos trabalhos foi através de arquivos de log. Os principais trabalhos que utilizaram essa abordagem para coleta foram (Schoor \& Bannert, 2012), (Sabourin, Mott, \& Lester, 2012), (Bondareva, et al., 2013), (Nussbaumer, Hillemann, Gütl, \& Albert, 2015), (Segedy, Kinnebrew, \& Biswas, 2015), (Sonnenberg \& Bannert, 2015), (You, 2016). A maioria destes trabalhos utiliza dados de softwares educacionais específicos para o e-Learning.

Um dos trabalhos merece destaque. É o trabalho do (You, 2016) que foi o único a utilizar arquivos de log provindos de ambientes de LMS. O estudo procurou identificar indicadores comportamentais para a aprendizagem autorregulada em 
sistemas de LMS para tentar prever o sucesso dos alunos ao termino de um curso. Para tal, utilizou a regressão linear para construção de modelos e verificação de variáveis significativas. O trabalho possui algumas limitações com relação a escolha das variáveis explicativas. Foram utilizadas apenas variáveis relacionadas a quantidade de acesso, tempos de visualizações e atraso na entrega de atividades. Estas variáveis não compõem nenhum dos modelos teóricos desenvolvidos na literatura para os construtos de SRL.

\subsection{Terceira questão de pesquisa}

A terceira questão de pesquisa deste mapeamento foi elaborada com o objetivo de identificar quais as principais técnicas que estão sendo utilizadas para analisar $e$ mensurar as características de SRL? As técnicas de análise mais utilizadas foram análise de regressão e análise fatorial, sendo quatro trabalhos que utilizaram regressão linear (Yukselturk \& Bulut, 2009), (Park \& Lim, 2015), (You, 2016) e quatro trabalhos que utilizaram análise fatorial confirmatória (Barnard, Lan, To, \& Paton, 2009), (Cho \& Jonassen, 2009), (Adesope, Zhou, \& Nesbit, 2015), (Ali, Hatala, Gašević, \& Winne, 2014).

A maioria dos trabalhos que utilizou a regressão linear como técnica principal tiveram como foco o desenvolvimento de modelos de previsão, especificamente o desempenho de alunos. Todos os trabalhos que utilizaram análise fatorial como técnica principal, tiveram como objetivo validar questionários ou a identificação de pesos ou cargas fatoriais dos construtos de SRL.

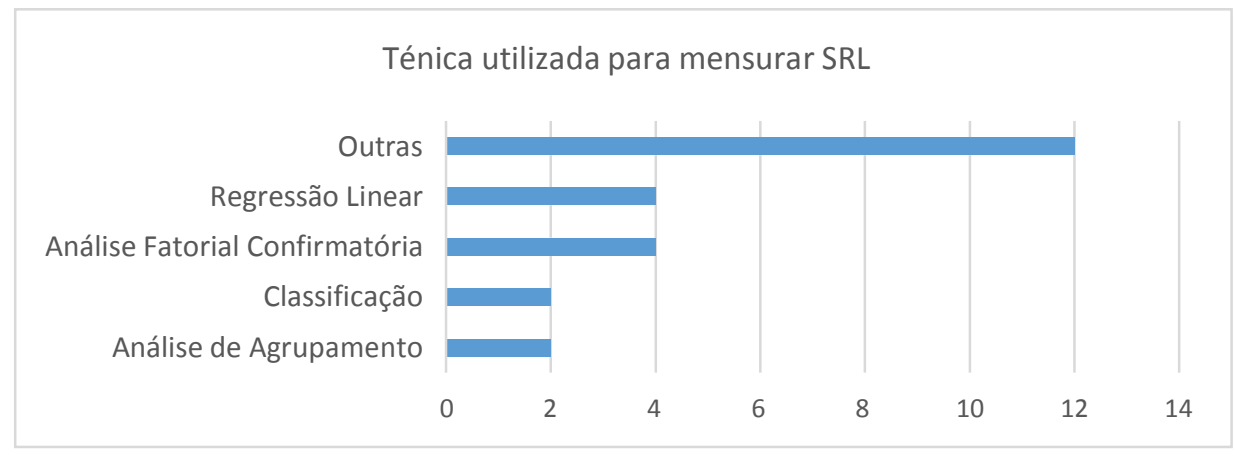

Figura 4: Técnica utilizada para mensuração de SRL.

Outras duas técnicas que foram utilizadas, foram a classificação e a análise de agrupamento, ambas com dois trabalhos encontrados na literatura. Os trabalhos de classificação (Bondareva, et al., 2013), (Sabourin, Mott, \& Lester, 2012) tiveram como foco o comparativo de múltiplos algoritmos de classificação de dados provindos de sistemas de tutores inteligentes e os trabalhos de análise de agrupamento (Lawanto, Santoso, Lawanto, \& Goodridge, 2014), (Colthorpe, Zimbardi, Ainscough, \& Anderson, 2015) tiveram o objetivo de identificar padrões ou perfis comportamentais de autorregulação.

\subsection{Quarta questão de pesquisa}

A quarta questão de pesquisa foi a identificação de quais as principais abordagens de pesquisa que dedicam esforços para o desenvolvimento e aplicação de técnicas de mensuração de SRL? A abordagem baseada em técnicas provindas da estatística foi a que teve o maior número de trabalhos, nos quais utilizaram técnicas como Testes de Comparação de Médias (Gašević, Adesope, Joksimović, \& Kovanović, 2015), (Segedy, Kinnebrew, \& Biswas, 2015), Análise Fatorial (Barnard, Lan, To, \& Paton, 2009), (Cho 
\& Jonassen, 2009), (Adesope, Zhou, \& Nesbit, 2015), (Ali, Hatala, Gašević, \& Winne, 2014), e Análise de Regressão Linear (Yukselturk \& Bulut, 2009), (Park \& Lim, 2015), (You, 2016).

Além desses, outros trabalhos utilizaram abordagens baseada em Mineração de Dados Educacionais, nos quais utilizam técnicas como Mineração de Processos (Schoor \& Bannert, 2012), (Sonnenberg \& Bannert, 2015), Análise de agrupamento (Lawanto, Santoso, Lawanto, \& Goodridge, 2014), (Colthorpe, Zimbardi, Ainscough, \& Anderson, 2015) e Algoritmos de Classificação (Bondareva, et al., 2013), (Sabourin, Mott, \& Lester, 2012).

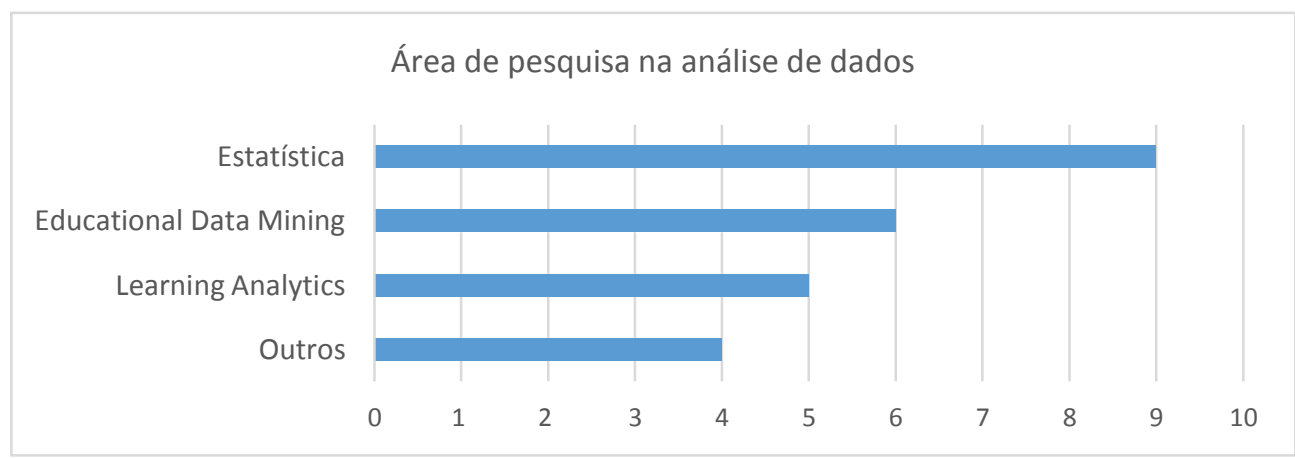

Figura 5: Área de pesquisa declarada na análise dos dados.

Outros quatro trabalhos utilizaram técnicas declaradas como sendo provindas da área de Learning Analytics. É importante mencionamos que uma mesma técnica pode ser considerada pertencer a mais de uma abordagem de pesquisa, como por exemplo a técnica de Análise de agrupamento, tem suas origens na Estatística, no entanto a área de mineração de dados e Learning Analytics tem evoluído e desenvolvido características algoritmos diferenciados para sua a aplicação.

A seção seguinte descreve as principais considerações obtidas com este mapeamento sistemático, bem como lacunas de pesquisa que serão abordados neste trabalho.

\section{Considerações Finais}

A análise dos trabalhos pesquisados e selecionados no mapeamento sistemático sobre os métodos utilizados para mensurar características de autorregulação da aprendizagem em ambientes de LMS, teve como objetivo identificar lacunas de pesquisa que subsidie e reforce futuras pesquisas na área.

Na primeira interação deste estudo observou-se que a área de pesquisa que busca entender o fenômeno de autorregulação da aprendizagem em ambientes de LMS vem aumentando ao longo dos anos, embora a grande maioria dos trabalhos encontrados na primeira interação tinha como abordagens de mensuração métodos qualitativos de análise. A segunda interação focou a busca por artigos que utilizassem apenas abordagens quantitativas de mensuração, dentro das áreas de estatística, mineração de dados, learning analytics e aprendizagem de máquina.

Um ponto que merece nos resultados encontrados é a predominância dois tipos de coleta de dados, as coletas baseadas em questionários e as coletas baseadas em arquivos de log. Os trabalhos que utilizaram questionário como instrumento principal de coleta, limitam-se a utilizar adaptações de questionários desenvolvidos especificamente 
para o ensino presencial, com exceção dos trabalhos que utilizaram o único questionário desenvolvido para aprendizagem em ambientes online.

Outra limitação refere-se à transposição dos modelos teóricos para as pesquisas com análise de dados. A literatura de SRL, no decorrer dos anos, vem desenvolvendo modelos teóricos e geração de construtos de autorregulação da aprendizagem, no entanto observamos que as pesquisas que buscam confirmar as características de SRL, através de análises quantitativas, não utilizam os construtos teóricos baseada nos modelos já desenvolvidos na literatura.

A grande maioria dos artigos que trabalharam com mensuração de desempenho, utilizaram questionários como o instrumento principal de coleta dos construtos de SRL. Apenas um dos trabalhos utilizou uma abordagem de coleta através de arquivos de log dos ambientes de LMS especificamente. No entanto este trabalho apresentou limitações no que diz respeito a seleção das variáveis que explicam os construtos de SRL. Foi utilizado apenas variáveis relacionadas a quantidade de acesso, tempos de visualizações e atraso na entrega de atividades. Estas variáveis não compõem nenhum dos modelos teóricos desenvolvidos na literatura para os construtos de SRL.

Não foi encontrado nenhum artigo com foco no desenvolvimento de soluções de software que deem suporte para professores ou tutores no que diz respeito a identificação de alunos com dificuldade de autorregulação.

Como trabalhos futuros pretendemos realizar o desenvolvimento de soluções de software que consumam dados comportamentais de autorregulação provindos de sistemas de LMS, como forma de identificar estados autorregulatórios de aprendizagem dos alunos e fornecer subsídios para professores e tutores a partir da análise de dados em tempo real.

\section{Agradecimentos}

Esta pesquisa é apoiada pela FACEPE-APQ No. 0525-1.03/14. Os autores também agradecem ao apoio da PRPPG/UFRPE (006999/2015-28) e PRPPGI/UNIVASF (Edital 18/2013). Alex Sandro Gomes é bolsista DT Nível 2/CNPq, processos n ${ }^{\circ} 310466 / 2012-$ 1 e no $475634 / 2013-6$.

\section{Referências}

ADESOPE, O. O., ZHOU, M., \& NESBIT, J. C. (2015). Achievement Goal Orientations and Self-Reported Study Strategies as Predictors of Online Studying Activities. Journal of Educational Computing Research, 436-458.

ALI, L., HATALA, M., GAŠEVIĆ, D., \& WINNE, P. H. (2014). Leveraging MSLQ Data for Predicting Students Achievement Goal Orientations. Journal of Learning Analytics, 157-160.

ARKSEY, H., \& O’MALLEY, L. (2005). Scoping Studies: Towards A Methodological Framework.

BARNARD, L., LAN, W. Y., TO, Y. M., \& PATON, V. O. (2009). Measuring self-regulation in online and blended learning environments. The Internet and Higher Education, 1-6.

BONDAREVA, D., CONATI, C., FEYZI-BEHNAGH, R., HARLEY, J. M., AZEVEDO, R., \& BOUCHET, F. (2013). Inferring learning from gaze data during interaction with an environment to support self-regulated learning. In Artificial Intelligence in Education , 229-238.

CHO, M. H., \& JONASSEN, D. (2009). Development of the human interaction dimension of the Self-Regulated Learning Questionnaire in asynchronous online learning environments. Educational Psychology, 117-138. 
COLTHORPE, K., ZIMBARDI, K., AINSCOUGH, L., \& ANDERSON, S. (2015). Know thy student! Combining learning analytics and critical reflections to develop a targeted intervention for promoting self-regulated learning. Journal of Learning Analytics, 134-15.

GAŠEVIĆ, D., ADESOPE, O., JOKSIMOVIĆ, S., \& KOVANOVIĆ, V. (2015). Externallyfacilitated regulation scaffolding and role assignment to develop cognitive presence in asynchronous online discussions. The Internet and Higher Education, 24, 53-65.

KITCHENHAM, B. (2007). Guidelines for performing Systematic Literature Reviews in . LAWANTO, O., SANTOSO, H. B., LAWANTO, K. N., \& GOODRIDGE, W. (2014). SelfRegulated Learning Skills and Online Activities between Higher and Lower Performers on a Web-Intensive Undergraduate Engineering Course. Journal of Educators Online.

MALMBERG, J., JÄRVELÄ, S., JÄRVENOJA, H., \& PANADERO, E. (2015). Promoting socially shared regulation of learning in CSCL: Progress of socially shared regulation among high-and low-performing groups. Computers in Human Behavior.

NUSSBAUMER, A., HILLEMANN, E. C., GÜTL, C., \& ALBERT, D. (2015). A competencebased service for supporting self-regulated learning in virtual environments. Journal of Learning Analytics, 101-133.

PANADERO, E., KIRSCHNER, P. A., JÄRVELÄ, S., MALMBERG, J., \& JÄRVENOJA, H. (2015). How Individual Self-Regulation Affects Group Regulation and Performance A Shared Regulation Intervention. Small Group Research.

PARK, Y., \& LIM, K. (2015). Effects of Environmental and Human Constructs on E-Learning Effectiveness in Online University Settings. Indian Journal of Science and Technology, 103109.

PETERSEN, K. E. (2007). Systematic Mapping Studies in Software Engineering.

ROLL, I., \& WINNE, P. H. (2015). Understanding, evaluating, and supporting self-regulated learning using learning analytics. Journal of Learning Analytics, 7-12.

SABOURIN, J. L., MOTT, B. W., \& LESTER, J. C. (2012). Early Prediction of Student SelfRegulation Strategies by Combining Multiple Models. International Educational Data Mining Society.

SANCHEZ-SANTILLAN, M., PAULE-RUIZ, M., CEREZO, R., \& ALVAREZ-GARCÍA, V. (2016). MeL: Modelo de adaptación dinámica del proceso de aprendizaje en eLearning. anales de psicología, 106-114.

SCHOOR, C., \& BANNERT, M. (2012). Exploring regulatory processes during a computersupported collaborative learning task using process mining. Computers in Human Behavior, 1321-1331.

SEGEDY, J. R., KINNEBREW, J. S., \& BISWAS, G. (2015). Using coherence analysis to characterize self-regulated learning behaviours in open-ended learning environments. 13-48.

SONNENBERG, C., \& BANNERT, M. (2015). Discovering the Effects of Metacognitive Prompts on the Sequential Structure of SRL-Processes Using Process Mining Techniques. Journal of Learning Analytics, 72-100.

YOU, J. W. (2016). Identifying significant indicators using LMS data to predict course achievement in online learning. The Internet and Higher Education, 23-30.

YUKSELTURK, E., \& BULUT, S. (2009). Gender differences in self-regulated online learning environment. Journal of Educational Technology \& Society, 12-22. 